\title{
RCT wins CMAJ Bruce Squires Award
}

$\mathrm{T}$

he authors of a randomized controlled trial looking at the weight of newborns whose mothers were immunized for influenza during pregnancy have been awarded for their contribution to health care research in Canada.

Dr. Mark Steinhoff and his international team of seven coauthors, including three from Bangladesh, are the recipients of the Bruce Squires Award for their article "Neonatal outcomes after influenza immunization during pregnancy: a randomized controlled trial" (CMAJ 2012;184:645-53).

Although the United States has recommended influenza immunization during pregnancy since 1960, this is the first evidence from a randomized controlled trial to support the wisdom of that policy. The trial, which involved 340 pregnant women in

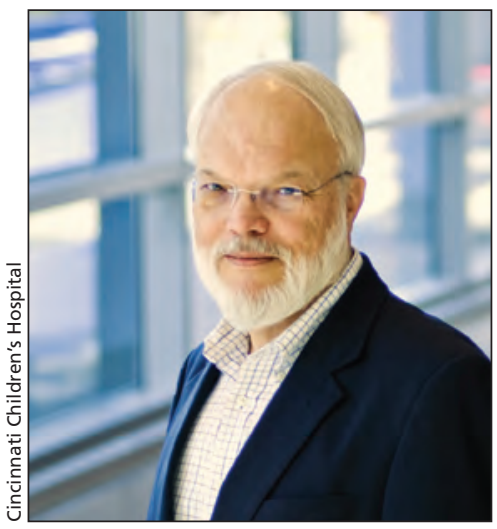

Dr. Mark Steinhoff and colleagues award-winning article has been highly cited. Bangladesh, showed that during flu season, maternal immunization during pregnancy was associated with a lower proportion of infants who were small for gestational age and an increase in mean birth weight by 200 grams.

"That was a completely new finding," says Steinhoff. "The vaccine clearly showed a difference in birth weight, indicating that maternal immunization benefited the fetus."

The women were all in their third trimester, a time when a fetus gains upwards of 30 grams per day. When the mother is ill and doesn't eat for a few days, it can make a big difference, says Steinhoff.

"The flu vaccine in pregnancy is a three-in-one," he adds. "It protects the mother, fetus and infant. All three benefit."

Since the study was published, several other researchers in the US and Canada have done secondary analyses of data and showed similar results.

Steinhoff is the director of the Global Health Center at the Cincinnati Children's Hospital Medical Center in Ohio and a professor of pediatrics at the University of Cincinnati College of Medicine. He coauthored the paper with Saad B. Omer, Eliza Roy, Shams El Arifeen, Rubhana Raqib, Caitlin Dodd, Robert F Breiman and K. Zaman, the senior author.

This award honours Editor Emeritus Dr. Bruce Squires, who served in various capacities at CMAJ from 1984 to 1996, including as editor-in-chief for seven years. Squires promoted high standards of reporting and evidence-based medicine. It is awarded annually to the author(s) of the research paper published in the journal that is most relevant to the practice of medicine and most likely to impact it in a positive way.

"This is a paper that Bruce Squires himself would have been proud to publish," says CMAJ Editor-in-Chief Dr. John Fletcher. "It's a randomized controlled trial, which is at the heart of high-quality evidence for evidence-based health care."

In selecting the winning paper, $C M A J$ considered the study's originality, potential to impact practice as well as its methodological rigour.

"This is a particularly good example of a trial that will make a difference to clinical practice," says Fletcher. In Canada, fewer than one in five pregnant women has had a recent flu shot, he points out. "Although this paper is about women in Bangladesh, it speaks directly to Canadians" health care and reassures Canadian women that the flu shot is good for them and their baby."

Fletcher adds that "I like to publish papers either at the beginning of the story, showing something for the first time - like this study — or at the end of the story, summarizing other people's research to give a definitive answer - like the systematic review that won last year" (CMAJ 2011;183:1359-66).

Steinhoff says he's "glad to see this work is being recognized. It was widely cited because it was the first to show this result. I do appreciate this award and it will be very meaningful to my colleagues in Bangladesh." - Barbara Sibbald, CMAJ 